\title{
P-Wave Dispersion and Its Relationship with the Severity of the Coronary Artery Disease in Patients with Stable Angina
}

Ahmed Kamal Metawea, Wael Mohamed Attia, Ahmed Abd Elraouf Mahdy*, Mohamed Gamal Salama

Cardiology Department, Al-Azhar University, Faculty of Medicine, Egypt

*Corresponding author: Ahmed Abd Elraouf Mahdy, Mobile: (+20) 01063635890, E-Mail: ahmedraoufcardiology@gmail.com

\begin{abstract}
Background: In Electrocardiography (ECG), the term "P-wave dispersion" (PWD) refers to variation between maximum and minimum P-waves recorded from various Electrocardiography leads. P-wave analysis in ECG may become more widely used marker in many clinical contexts as a result of improved methods for capturing and analyzing this ECG. The link between (PD) and severity of coronary artery disease (CAD) was established among personnel who had stable form of CAD. Objective: We wanted to determine if there was an association between $\mathrm{P}$ wave dispersion and CAD severity in patients who had stable CAD.

Patients and methods: At Al-Hussein University Hospital Cath Lab, 51 consecutive patients were referred for diagnostic coronary angiography in a cross-sectional trial. They were classified into group A (control group, $n=8$ ), who were found to have normal coronary angiography and group B $(n=43)$, who were found to have coronary artery disease. Results: $\mathrm{P}$ wave dispersion was significantly different across cases, with 95.3 percent of patients having abnormal $\mathrm{P}$ wave dispersion compared to 100 percent of control cases. P wave dispersion and Gensini score were statistically significantly different between cases and controls where cases had significantly higher scores than controls. Dispersion of $\mathrm{P}$ waves was statistically linked to number of vessels affected as well as Gensini scores. The $\mathrm{P}$ wave dispersion and the Gensini score showed a strong positive association.

Conclusion: Stable coronary artery disease (CAD) patients had aberrant PWD, according to the findings of this study, in patients with stable coronary artery disease, severity of the CAD is correlated with $\mathrm{P}$ wave dispersion.
\end{abstract}

Keywords: CAD, Stable Angina, P-wave dispersion.

\section{INTRODUCTION}

Coronary artery disease, often known as atherosclerotic coronary heart disease or ischemic heart disease (IHD), is a group of conditions that includes stable and unstable angina, sudden coronary death, and myocardial infarction (MI). Mortality and morbidity are more likely to occur as a result of these conditions ${ }^{(\mathbf{1})}$.

In Electrocardiography (ECG), the term "P-wave dispersion" (PD) refers to variation between maximum and minimum $\mathrm{P}$-waves recorded from various Electrocardiography leads. P-wave analysis in ECG may become more widely used marker in many clinical contexts as a result of improved methods for capturing and analyzing this ECG specially for determination of Atrial Fibrillation (AF) risks ${ }^{(2)}$. Many researches were done to study P- wave dispersion among conditions of cardiac patients like dilated cardiomyopathy, coronary angioplasty or angina pectoris, hypertension, diabetes mellitus, obesity and myocardial infarction ${ }^{(3)}$.

Akin et al. (4) studied people who had stable coronary artery disease and found that the illness's severity was linked to an elevated PD level.

We aimed at this trial to study if personnel who had stable coronary artery disease were found to have a correlation among $\mathrm{P}$ wave dispersion and CAD severity.

\section{PATIENTS AND METHODS}

At Al-Hussein University Hospital Cath Lab, 51 consecutive patients were referred for diagnostic coronary angiography in a cross-sectional trial. They were classified into group A (control group, $n=8$ ), who were found to have normal coronary angiography and group B $(n=43)$, who were found to have CAD. At Al Hussein University Hospital's Cath lab, the patients underwent coronary angiography as part of this study to determine if they had coronary artery disease.

Inclusion criteria: Stable coronary artery disease patients with chest discomfort or other symptoms that warranted coronary angiography.

\section{Exclusion criteria:}

- Atrial fibrillation with an invisible P wave.

- Acute coronary syndromes (ACS).

- Cardiomyopathy.

- Antiarrhythmic medications use.

- Patient that has a history of past heart attacks and heart surgery.

- P-wave analysis might be hindered by any irregularities in the heartbeat (frequent atrial and ventricular ectopic, pacemaker rhythm).

- Altered functions of thyroid.

- Disturbance in serum electrolytes

\section{Ethical consent:}

An approval of the study was obtained from AlAzhar University Academic and Ethical Committee. Every patient signed an informed written consent for acceptance of the study. This work has been carried out in accordance with The Code of Ethics of the World Medical Association (Declaration of Helsinki) for studies involving humans.

All patients were subjected the followings: 


\section{Complete history taking and physical examination:}

With focus on age, sex, and a history of risk factors of CAD such like high blood pressure (BP $140 / 90 \mathrm{mmHg}$ or higher), diabetes mellitus, smoking, and family history of IHD, chest pain, previous myocardial infarction, and previous catheterization or revascularization.

\section{Electrocardiography:}

Supine patients were subjected to a 12-lead electrocardiogram (ECG). Speed of $50 \mathrm{~mm} / \mathrm{s}$ and $1 \mathrm{mV}$ per $\mathrm{cm}$ standardization were used to record the data. ECG reporting without knowing the patient's clinical state. There were two P-wave lengths physically measured, and the difference between $\mathrm{P}$ max and $\mathrm{P}$ min is defined as $\mathrm{P}$ Dispersion (PD) was determined. Magnifying lenses were utilized to improve the precision of the calipers we employed. More than ten leads of an ECG with discernible $P$ waves were included in this study. ${ }^{(5)}$

\section{Assessment by echocardiography:}

Echocardiogram was done for all patients. Available echocardiographic equipment was used (Hewlette Packard) Sonos 5500 and Philips system both equipped with DTI technology. All patients had an Mmode, 2-D, and Doppler echocardiographic evaluation. Patient was positioned semi-laterally in the left semilateral position for examinations, which included views of the left parasternal long and short axes, as well as apical 4 and 2 chambers. The American Society of Echocardiography's guidelines were followed in the recording and calculation of data.

2D-guided M-mode was commonly used to estimate the left ventricular dimensions from parasternal projections, following the leading edge to leading edge convention. The left atrium and left ventricle were measured, the ejection fraction (EF) of the left ventricular system was assessed as well as the presence of mitral insufficiency. ${ }^{(6)}$

\section{Coronary angiography:}

When the Gensini score was used to guide the procedure, it was possible to determine the severity of the CAD. Percutaneous transfemoral method was used to complete the procedure (Judkins technique). A Judkins left catheter was used for left coronary angiography, where a Judkins or Amplatz right catheter was used for right coronary side. Gensini scoring system classified the narrowing of coronary arteries' lumen as 1 for a 1 to 25 per cent, 2 for a $26-50$ per cent, 4 a 51-75 percent, 8 a $76-90$ percent, 16 a $91-99$ percent, and 32 for total occlusion. It is then divided by a factor that indicates how important the lesion's location is to the score. Lesion of the left main system multiplied by 5 it is 2.5 for lesions in the LAD and $\mathrm{Cx}, 1.5$ for those in the middle of the LAD, and 1 for those in the right coronary artery, the mid/distal CX, and the proximal LAD. 0.5 is the multiplicand for all other branches. ${ }^{(7)}$

Statistical analysis
Microsoft Excel was used throughout the patient's history, clinical examination, and laboratory tests. Statistical Package for the Social Sciences (SPSS) was used to further analyze the information. The Chisquare test was used to evaluate group percentages and frequencies (qualitative variables) to see if the differences between groups are statistically significant. In order to compare parametric quantitative independent groups, Kruskal Wallis's ANOVA was used. Man Whitney's $t$ test was utilized for non-parametric. Pearson and Spearman for correlated data were available. For significant results, the p-value was set at 0.05 and 0.001 for extremely significant results.

\section{Results:}

Fifty-one cases participated in this study and were classified into: Group A $(\mathrm{n}=8)$, patients who were found to have normal coronary angiography, and Group B $(n=43)$, patients who were found to have coronary artery disease.

Patients with coronary artery disease ware classified according to number of vessels: One vessel 18 patients, two vessel 12 patients, and three vessel 13 patients.

Table (1) showed that the mean age of total study population was $59.29 \pm 8.79$ with minimum age of 39 and maximum age of 76 years. Regarding sex distribution males represented $60.8 \%$ and females represented $39.2 \%$.

Table (2) showed that DM patients represented $80.4 \%$ from studied participants, hypertension (HTN) represented $76.5 \%$ and hyperlipidemia represented $82.4 \%$.

Table (3) showed that $15.7 \%$ of participants had no significant CAD. The majority of cases were one vessel affection as they represented $35.3 \%$ from total followed by three vessels and two vessels $25.5 \%$ and $23.5 \%$ respectively. Regarding P wave dispersion $80.4 \%$ was abnormal (> 0.04).

Table (4) showed the distribution of EF as it was 57.35 \pm 6.85 , Gensini score was $27.05 \pm 26.4$ and $\mathrm{P}$ wave dispersion was $0.073 \pm 0.025$.

\section{Comparison between cases and control:}

Table (5) showed correlation of $\mathbf{P}$ wave dispersion between cases and control, which showed statistically significant difference of abnormal $\mathrm{P}$ wave dispersion regarding cases as $95.3 \%$ of cases had abnormal $\mathrm{P}$ wave dispersion, while $100 \%$ of control with normal $P$ wave. Table (6) Comparison between cases and control regarding EF, Gensini score and $P$ wave dispersion showed statistically significant difference between cases as Gensini score and $\mathrm{P}$ wave dispersion were significantly higher in cases than in control.

Table (7) Comparison among different vessels affected regarding EF, according to the results of the Gensini score and $\mathrm{P}$ wave dispersion, the three-vessel group had a considerably lower EF compared to both of the other two groups. However, there was no statistically significant difference between the other two groups when it came to EF. 
Table (8) showed that correlations between P wave dispersion and Gensini scores as well as the number of vessels impacted were statistically significant.

Table (1): Distribution of participants in terms of gender and age

\begin{tabular}{|l|c|}
\hline Age (yrs) & $59.29 \pm 8.79$ \\
\hline Male gender & $31(60.8 \%)$ \\
\hline
\end{tabular}

Table (2): Baseline characters

\begin{tabular}{|l|c|c|}
\hline & NO & YES \\
\hline DM & $10(19.6 \%)$ & $41(80.4 \%)$ \\
\hline HTN & $12(23.5 \%)$ & $39(76.5 \%)$ \\
\hline Hyperlipidemia & $9(17.6 \%)$ & $42(82.4 \%)$ \\
\hline Total & \multicolumn{2}{|c|}{$\mathbf{5 1}$} \\
\hline
\end{tabular}

Table (3): Number of vessels and $\mathrm{P}$ wave dispersion

\begin{tabular}{|c|c|c|}
\hline & & N\% \\
\hline \multirow{4}{*}{ Number of Vessels } & $\mathrm{NO}$ & $8(15.7 \%)$ \\
\hline & One Vessel & $18(35.3 \%)$ \\
\hline & Two Vessels & $12(23.5 \%)$ \\
\hline & Three Vessels & $13(25.5 \%)$ \\
\hline \multirow[t]{2}{*}{ P wave dispersion } & Normal & $10(19.6 \%)$ \\
\hline & Abnormal & $41(80.4 \%)$ \\
\hline Total & \multicolumn{2}{|c|}{51} \\
\hline
\end{tabular}

Table (4): Distribution of EF, Gensini score and P wave dispersion

\begin{tabular}{|l|c|}
\hline & Mean \pm SD \\
\hline EF & $\mathbf{5 7 . 3 5} \pm 6.85$ \\
\hline Gensini score & $\mathbf{2 7 . 0 5} \pm \mathbf{2 6 . 4}$ \\
\hline P wave dispersion & $\mathbf{0 . 0 7 3} \pm 0.025$ \\
\hline
\end{tabular}

Table (5): $\mathrm{P}$ wave dispersion between cases and control

\begin{tabular}{|l|l|c|c|c|}
\hline \multicolumn{2}{|c|}{} & \multicolumn{2}{|c|}{ Groups N (\%) } & \multirow{2}{*}{ P } \\
\cline { 3 - 4 } \multirow{2}{*}{ Wave } & Normal $\leq 0.04$ & Control $(\mathbf{N}=\mathbf{8})$ & Cases $(\mathbf{N}=\mathbf{4 3})$ & \\
\cline { 2 - 4 } & Abnormal $>0.04$ & $\mathbf{8}(\mathbf{1 0 0 . 0 \%})$ & $\mathbf{2}(\mathbf{4 . 7 \%})$ & \multirow{2}{*}{$\mathbf{0 . 0 0} * *$} \\
\hline
\end{tabular}

Table (6): Comparison between cases and control regarding EF, Gensini score and $\mathrm{P}$ wave dispersion

\begin{tabular}{|c|c|c|c|c|c|c|}
\hline & Groups & $\mathbf{N}$ & Mean & $\begin{array}{c}\text { Std. } \\
\text { Deviation }\end{array}$ & $\begin{array}{c}\text { t/Man } \\
\text { Whitney }\end{array}$ & $\mathbf{P}$ \\
\hline \multirow[t]{2}{*}{$\mathbf{E F}$} & Cases & 43 & 56.72 & 6.558 & \multirow[t]{2}{*}{-1.548} & \multirow[t]{2}{*}{0.128} \\
\hline & Control & 8 & 60.75 & 7.851 & & \\
\hline \multirow[t]{2}{*}{ Gensini score } & Cases & 43 & 32.09 & 25.8 & \multirow[t]{2}{*}{5.489} & \multirow[t]{2}{*}{$0.01 * *$} \\
\hline & Control & 8 & 0.00 & 0.0 & & \\
\hline \multirow{2}{*}{$P$ wave dispersion } & Cases & 43 & 0.08 & 0.022 & \multirow[t]{2}{*}{6.920} & \multirow[t]{2}{*}{ 0.01** } \\
\hline & Control & 8 & 0.04 & 0.0 & & \\
\hline
\end{tabular}

Table (7): Comparison among different vessel affected groups regarding EF, Gensini score and P wave dispersion

\begin{tabular}{|c|c|c|c|c|c|c|c|}
\hline & $\mathbf{N}$ & Mean & SD & Minimum & Maximum & $\mathbf{P}$ \\
\hline \multirow{3}{*}{$\mathbf{E F}$} & One vessel & 18 & 57.79 & 5.67 & 48.00 & 69.00 & \multirow[t]{3}{*}{ 0.038* } \\
\hline & Two vessels & 12 & 59.41 & 6.92 & 48.00 & 71.00 & \\
\hline & Three vessels & 13 & 53.07* & 6.19 & 40.00 & 65.00 & \\
\hline \multirow[t]{3}{*}{ Gensini score } & One vessel & 18 & 15.22 & 19.28 & 4.00 & 90.00 & \multirow[t]{3}{*}{$0.01 * *$} \\
\hline & Two vessels & 12 & 35.83 & 21.78 & 11.00 & 86.00 & \\
\hline & Three vessels & 13 & 52.00 & 22.31 & 18.00 & 92.00 & \\
\hline \multirow{3}{*}{$\begin{array}{l}\text { P wave } \\
\text { dispersion }\end{array}$} & One vessel & 18 & 0.061 & 0.01 & 0.04 & 0.08 & \multirow[t]{3}{*}{$0.01 * *$} \\
\hline & Two vessels & 12 & 0.086 & 0.021 & 0.06 & 0.12 & \\
\hline & Three vessels & 13 & 0.10 & 0.016 & 0.08 & $\mathbf{0 . 1 2}$ & \\
\hline
\end{tabular}


Table (8): Correlations between $\mathrm{P}$ wave dispersion and age, EF Gensini score and vessel score

\begin{tabular}{|c|c|c|}
\hline - & & $P$ wave dispersion \\
\hline \multirow[t]{2}{*}{ Age } & $\mathrm{r}$ & 0.171 \\
\hline & $\mathrm{P}$ & 0.272 \\
\hline \multirow[t]{2}{*}{$\mathbf{E F}$} & $\mathrm{r}$ & -0.185 \\
\hline & $\mathrm{P}$ & 0.236 \\
\hline \multirow[t]{2}{*}{ Gensini score } & $\mathrm{r}$ & 0.766 \\
\hline & $\mathrm{P}$ & $0.00 * *$ \\
\hline \multirow[t]{2}{*}{ Number of Vessel } & $\mathrm{r}$ & 0.721 \\
\hline & $\mathrm{P}$ & $0.00 * *$ \\
\hline
\end{tabular}

\section{DISCUSSION}

Myocardial infarction, obesity, peripheral vascular disease, hypertension, and diabetes are all associated with a higher risk for persons with a higher PD, according to Karabag et al. ${ }^{(15)}$ and Yilmaz and Demirbag (16) who found apparent association between $\mathrm{P}$ wave dispersion and the presence of stable coronary artery condition.

$\mathrm{P}$ wave dispersion and coronary artery disease severity were examined in this study, which aimed to examine the link between these two variables. The mean age was $59.29 \pm 8.79$ with minimum age of 39 and maximum age of 76 years. Regarding sex distribution males represented $60.8 \%$ and females represented $39.2 \%$. We could not discern an age or gender difference between cases and controls.

Also, DM patients represented $80.4 \% \%$ from studied participants, HTN represented $76.5 \%$, and hyperlipidemia represented $82.4 \%$. In terms of diabetes and hypertension, there was no statistically significant difference between the patients and the control groups. Cases had greater levels of hyperlipidemia than controls, although there was no statistically significant difference.

Findings from this research showed a link to severe coronary artery disease in those with PWD. P max, PD were likewise increased in the CAD group according to Akin et al. ${ }^{(8)}$. In all four patient' groups, PD was significantly higher than in the control group, regardless of vascular score.

When comparing cases and controls, we found that the Gensini score and $\mathrm{P}$ wave dispersion were both statistically substantially higher in the cases.

In our study, it was found that three vessel group had significantly lower EF than other two groups with no significant difference between the other two groups. Also, there was statistically significant difference between the three groups regarding Gensini score and $\mathrm{P}$ wave dispersion as three vessel group were significantly higher followed by two vessel group.
Dilaveris et al. ${ }^{(2)}$ found in their trial which contained confirmed $95 \mathrm{CAD}$ patients that myocardial ischemia prolongs PD. Angina patients with myocardial ischemia were found to have a higher PD, according to Ozmen and colleagues ${ }^{(3)}$. There was a link between PD and carotid artery atherosclerosis. With coronary slow-flow phenomena, it has been found that P-wave dispersion was enhanced.

$\mathrm{P}$ wave dispersion and Gensini and vessel scores were found to have substantial positive associations in our investigation. P max and PD were showed to be linked to Gensini scores among CAD group, according to Akin $\boldsymbol{e t}$ al. ${ }^{(4)}$. Increased PD was found to be linked with a greater number of afflicted vessels and a higher Gensini score in multivariate logistic regression analysis.

When it comes to PWD, Dogan and colleagues ${ }^{(8)}$ investigated the link between coronary artery ectasia (CAE) and the PWD condition. There were 26 patients (average age $61.6 \pm 11.0$ years) with isolated CAE and 26 control participants in their study sample (group 2). Because CAE is a kind of coronary atherosclerosis, it can lead to atrial ischemia, which in turn can lead to ventricular fibrillation (VF). There is an association between the number of ectatic arteries in people with CAE and the prevalence of PWD.

An investigation of the connection between PWD and CAD severity was conducted by Akin $\boldsymbol{e t}$ al. ${ }^{(4)}$. 60 patients with severe $(50 \%)$ coronary stenosis; categorized into a control group who had normal heart angiogram, and a case group, those who had coronary artery disease. In individuals with stable coronary artery disease (CAD), PWD was shown to be higher than in controls and to be linked with disease severity. The Gensini score was revealed to be an independent predictor of PWD in multiple regression analysis.

In individuals with stable coronary artery disease, Akin et al. ${ }^{(4)}$ investigated the relationship between PD and the severity of coronary artery disease. They found that PD was higher in patients 
with CAD than in controls, and it was linked with the severity of the CAD.

\section{CONCLUSION}

In individuals with stable coronary artery disease, this study found that $\mathrm{P}$ wave dispersion is associated with the severity of CAD.

\section{Financial support and sponsorship: Nil.} Conflict of interest: Nil.

\section{REFERENCES}

1. Mehta P, Wei J, Wenger $\mathbf{N}$ (2015): Ischemic heart disease in women: a focus on risk factors. Trends Cardiovasc Med., 25 (2): 140-51.

2. Dilaveris $\mathbf{P}$, Gialafos $\mathbf{J}$ (2001): P-wave dispersion: a novel predictor of paroxysmal atrial fibrillation. Ann Noninvasive Electrocardiol., 6 (2): 159-65.

3. Özmen F (2001): Effect of balloon-induced acute ischaemia on $\mathrm{P}$ wave dispersion during percutaneous transluminal coronary angioplasty. Europace., 3 (4): 299-303.

4. Akin F, Firatli I, Katkat F et al. (2014): P-wave dispersion and its relationship with the severity of the disease in patients with stable coronary artery disease. North Clin Istanbul, 1 (2): 65-70.

5. Kose S, Aytemir K, Can I et al. (2002): Seasonal variation of $\mathrm{P}$-wave dispersion in healthy subjects. J Electrocardiol., 35 (4): 307-11.

6. Lang R, Badano L, Mor-Avi V et al. (2015): Recommendations for Cardiac Chamber Quantification by Echocardiography in Adults: An Update from the American Society of Echocardiography and the European Association of Cardiovascular Imaging. $\mathrm{J}$ Am Soc Echocardiogr., 28 (1): 1-39.

7. Gensini G (1975): Coronary Arteriography Coronary Arteriography. Mount Kisco, N. Y., Futura Publishing Co., Pp: 488. Available from: https://catalogue.nla.gov.au/Record/362970
8. Dogan S, Yildirim N, Aydin M et al. (2008): Assessment of P-wave duration and dispersion in patients with isolated coronary artery ectasia. Int $\mathrm{J}$ Cardiol., 125 (3): 404-6.

9. Cha Y, Dzeja P, Shen W et al. (2003): Failing atrial myocardium: energetic deficits accompany structural remodeling and electrical instability. Am J Physiol Circ Physiol., 284 (4): 1313-20.

10. Tükek T, Akkaya V, Demirel St et al. (2000): Effect of Valsalva maneuver on surface electrocardiographic P-wave dispersion in paroxysmal atrial fibrillation. Am J Cardiol., 85 (7): 896-9.

11. Gunduz H, Akdemir R, Binak E et al. (2003): Relation between stage of left ventricular diastolic dysfunction and QT dispersion. Acta Cardiol., 58 (4): 303-8.

12. Yilmaz $R$, Demirbag $R$ (2005): P-wave dispersion in patients with stable coronary artery disease and its relationship with severity of the disease. J Electrocardiol., 38 (3): 279-84.

13. Tsioufis C, Syrseloudis D, Hatziyianni A et al. (2010): Relationships of $C R P$ and $P$ Wave Dispersion With Atrial Fibrillation in Hypertensive Subjects. Am J Hypertens., 23 (2): 202-7.

14. Tsai W, Lee K, Kuo H et al. (2013): Association of increased arterial stiffness and $p$ wave dispersion with left ventricular diastolic dysfunction. Int J Med Sci., 10 (11): 1437-44.

15. Karabag T, Dogan S, Aydin M et al. (2012): The value of $P$ wave dispersion in predicting reperfusion and infarct related artery patency in acute anterior myocardial infarction. Clin Invest Med., 35: 12-9.

16. Yilmaz $R$, Demirbag $R$ (2015): P-wave dispersion in patients with stable coronary artery disease and its relationship with severity of the disease. J Electrocardiol., 38: 279-84. 